\title{
A Review of Novel Formulation Strategies to Enhance Oral Delivery of Zaleplon
}

\section{Narendar Dudhipala*}

Department of Pharmaceutics, Vaagdevi Institute of Pharmaceutical Sciences, Warangal, Telangana-506009, India

\begin{abstract}
Zaleplon is a pyrrazolopyrimidine which is recommended for the treatment of insomnia and pentylenetetrazole/ electroshock-induced convulsions is an effective anticonvulsant which potential acts on the GABA receptor. The poor oral bioavailability of zaleplon $(\sim 30 \%)$ is due to the low solubility which in turn amputates the dissolution of the drug, restricts gastric absorption and first-pass metabolism. It is now withdrawn from the market due to the high dose and inefficient therapeutic activity. This review focuses on the various drug systems of zaleplon such as solid dispersions, self-nanoemulsifying, solid dispersions, proliposomes and solid lipid nanoparticles to enhance the oral bioavailability.
\end{abstract}

Keywords: Zaleplon; Oral bioavailability; Dissolution; Proliposomes, Solid lipid nanoparticles

\section{Introduction}

Solubility is one of the major limitations in dosage form development of BCS class II and IV pharmaceutical actives. Majority of the new and existing active pharmaceutical ingredients fall in this class possessing low rate of dissolution and/or poor capacity to permeate across the biological membranes amputating their oral bioavailability. Copious techniques like cyclodextrin inclusion complexes; derivatization of the drug by strong or weak electrolytes; particle size reduction of the API using stabilizers and manipulating the drug from its crystalline form to amophorus form using suitable vehicles were investigated by formulation scientists belonging to various research groups to not only improve dissolution of the poorly soluble API but also enhance the bioavailability of lipophilic or hydrophobic drugs. Alternate drug delivery routes like transdermal and intranasal routes were also explored to enhance the bioavailability of drugs with either low solubility or permeability issues [1-9].

Zaleplon, a pyrazolopyrimidine hypnotic drug, is prescribed for the treatment of in insomnia and is also a potential anticonvulsant which minimizes the convulsions induced by phenylenetetrazoleand electroshock [10]. Following administration of zaleplon via oral route the drug is rapidly absorbed, however its absorption is limited due to its poor dissolution owing to its poor aqueous solubility and further delays the onset of action. In conjunction, the bioavailability is only $30 \%$ because of extensive first pass metabolism [11]. The major disadvantage of zaleplon is high dose and also causes for occurrence of (typically short-lived) hallucinations. Hence, various approaches have been adopted to enhance the bioavailability of zaleplon by improving its solubility which in turn improves the dissolution rate. These techniques in addition also aided to avoid first-pass metabolism. This review mainly deals with the various approaches mainly solid dispersions, complexation with cyclodextrins; lipid based delivery systems such as proliposomes, nanoemulsifying powders, semisolid dispersions with lipid surfactants and solid lipid nanoparticles to improve the oral bioavailability.

\section{Solid Dispersions of Zaleplon}

Initially, Waghmare et al. [12] reported to use solid dispersion technique to enhance the rate of dissolution of zaleplon. The solid dispersions were formulated using various hydrophilic polymers such as Leutrol F68, PVP k30 and PEG 6000 with the aid of solvent evaporation method. The solid-state properties of binary systems were characterized by powder XRD and DSC, with increase in the proportion of the polymer a gradual reduction in the crystalline nature of the drug in the binary systems of zaleplon was observed. The results from the study infer that the zaleplon dissolution can be improved to a greater extent by using solid dispersion techniques like solvent method. In addition the results from these studies conclude that, Solid dosage forms containing zaleplon for oral administration when formulated with poloxamer F68, PVP K30 or PEG 6000 improves the dissolution rate and leads to improvement in the bioavailability of the API to some extent.

Popescu et al. reported the solubility enhancement of zaleplon using complexation technique with cyclodextrin and modified cyclodextrins mainly methyl- $\beta$-cyclodextrin, hydroxypropyl- $\beta$-cyclodextrin and sulfobutylether- $\beta$-cyclodextrin. The solid dispersions are prepared by freeze drying and spray drying methods. Solid state characterization studies reveal the amorphous form of inclusion complex and are the reason for enhancement of solubility of drug. Also reported that solid dispersions made with hydroxypropyl- $\beta$-cyclodextrin and sulfobutylether- $\beta$-cyclodextrin shows superior solubility of drug than methyl- $\beta$-cyclodextrin [13].

Dudhipala et al. reported the a semisolid dispersion (SSD) of zaleplon when formulated using novel self-emulsifying lipid based amphiphilic carriers like TPGS E or Gelucire 44/14, resolve the issues associated with poor solubility of zaleplon [14]. It is quite evident from the phase solubility studies that a linear relationship was found between the drug solubility and concentration of lipid surfactant in aqueous medium which resulted in an $A_{L}$ type phase diagram. Fusion method when used to formulate zaleplon semisolid dispersions showed a high content uniformity of drug. The microscopic images obtained from the studies using a polarized optical microscopic studies revealed a change

*Corresponding author: Narendar Dudhipala, Associate Professor, Vaagdev Institute of Pharmaceutical Sciences, Warangal, Telangana-506009, India, Tel: 8099722523; E-mail: dnrlu14@gmail.com

Received July 06, 2016; Accepted July 15, 2016; Published July 22, 2016

Citation: Dudhipala N (2016) A Review of Novel Formulation Strategies to Enhance Oral Delivery of Zaleplon. J Bioequiv Availab 8: 211-213. doi:10.4172/jbb.1000297

Copyright: (c) 2016 Dudhipala N. This is an open-access article distributed under the terms of the Creative Commons Attribution License, which permits unrestricted use, distribution, and reproduction in any medium, provided the original author and source are credited. 
Citation: Dudhipala N (2016) A Review of Novel Formulation Strategies to Enhance Oral Delivery of Zaleplon. J Bioequiv Availab 8: 211-213. doi:10.4172/jbb.1000297

in crystalline form of drug to amorphous or molecular state. The loftier dissolution parameters of zaleplon from SSD over pure crystalline drug obtained from in vitro dissolution studies clearly predict the ability of these lipid surfactants as solubility enhancers. Further, a higher human effective permeability coefficient was observed which encourages the GI absorption of drug from SSD in situ intestinal permeation study was observed when using TPGS E or Gelucire 44/14. The in vivo studies carried out using male wister rats also demonstrated an improvement in the oral bioavailability of zaleplon from SSD over control pure drug suspension signifying the competence of Gelucire 44/14 (2.68 \pm 1.15 folds) and TPGS E (3.21 \pm 0.91 folds) as potential carriers that improve the rate of dissolution and also improve the limited bioavailability of zaleplon.

The significant enhancement in the zaleplon bioavailability from the formulations of ZST4 and ZSG4 can be related to many factors which either in combination or alone contribute for enhancement of the absorption by a magnitude. One of the potential factors that may be a key for the improvement in the bioavailability of zaleplon may be because of the betterment in the availability of the drug at the site of administration. The various reasons that account for the improvement of bioavailability may also be because of the formation of micelles that improve the effective surface area and further enhance the diffusion through the aqueous filled channels present within the cell membrane structure. Additionally, these self-emulsifying amphiphilic surfactants can either alter the barrier properties of GI membrane by fluidization of membrane or modfiy the efflux by acting as a P-gp activity modulator and hence, promoting the partitioning of the drugs into the bilayer facilitates the absorption $[15,16]$. In conclusion from the results of these studies it is evident that the self-emulsifying lipid surfactants act as potential carriers in enhancing the dissolution and oral bioavailability of BCS class II drug, zaleplon.

\section{Self-Nenoemulsifying Delivery of Zaleplon}

Karthik et al., 2013 reported the use of lipid emulsion based delivery systems for the enhancement of zaleplon oral bioavailability with the aid of self-nanoemulsifying systems. When formulating the emulsifying systems they first the developed the zaleplon nanoemulsions and converted the nanoemulsions to a powder form by adsorbing it onto different adsorbents like maltodextrin, aerosil 200 , pearlitol SD200, neusilin US2. From the preliminary studies it was evident that Neusilin US2 because of its high specific area and unique oil adsorption capacity acts as a better absorbent and was selected as adsorbent. A higher predicted permeability coefficient and percentage fraction oral dose was absorbed from the in situ rat intestinal perfusion studies for self-nanoemulsifying powder formulation with respect to control indicates that the capability of the drug to permeate across the intestinal barrier was enhanced. An in vivo pharmacokinetic study was conducted in Wister rats administering a suspension at a control dose of $10 \mathrm{mg} / \mathrm{kg}$. The results from the PK studies revealed an enhancement of $\sim 3$ fold in the oral bioavailability upon the administration of nano formulations compared to a suspension. The authors also proposed the possible mechanisms that are responsible for the enhancement of drug bioavailabilty of the nano powders which either in combination or alone contribute for enhancement of absorption by an order of magnitude. One of the key factors for hike in bioavailability of zaleplon is because of the better presentation of the drug at the site of administration. The reasons that can explicit include: (i) The remarkable increase in the surface area which is due to the formation of nanosized emulsion droplets, (ii) Diffusion of fine sized nano droplets via the aqueous filled channels that are present in the cell membrane structure, (iii)
Alteration of the barrier properties of GI membrane using surfactants, (iv) The promotion of drug partitioning due to the presence of oil and amphiphilic surfactants that facilitates drug absorption, and (v) The reduction of gastric emptying rate because of the long-chain lipids.

\section{Proliposomes}

Janga et al. [17] formulated proliposomes for enhanced oral bioavailability of zaleplon and also investigated the role of surface charge on the enhancement of zaleplon bioavailability. Hydrogenated soyphosphatidylcholine and cholesterol in varying ratios were used to formulate the proliposomes, and the finalized formulation was tailored with dicetyl phosphate to obtain negative charge vesicles and stearylamine to attain positive charged vesicles, respectively. Film disposition method was adopted to formulate the zaleplon proliposomes with the aid of spray dried mannitol as a carrier. The proliposomes were characterized for micromeritics, size, zeta potential, and entrapment efficiency. In vitro release and dissolution studies were also carried out and the results from these studies provide a clear insight on the enhanced dissolution of zaleplon and stability from proliposome formulations. The formulations were also characterized using solid state characterization techniques like SEM, DSC, and PXRD studies to investigate the conversion of zaleplon to amorphous or molecular state from its native crystalline form. In situ single-pass perfusion and bioavailability studies were also performed in rats to further confirm the conversion of the crystalline drug to amorphous form. The effective permeability coefficient (Peff) and the rate and extent of absorption from cationic vesicles was significantly enhanced and this enhancement is a clear indication that surface charge plays a major role in enhancing the effective uptake of drugs across the gastrointestinal tract. An enhancement of two- to fivefold be observed upon the use of proliposoems and this enhancement in bioavailability clearly indicates that proliposoems are potential carriers that enhance the oral bioavailability of zaleplon when administered via oral route [18-20].

\section{Conclusion}

Multiple formulation approaches to improve the bioavailability of the poorly soluble drugs are discussed in this article. Similar approaches using formulation of solid dispersions via spray drying, freeze drying or techniques like self nanoemulsifying systems or proliposomal systems can be considered to be potential strategies to enhance the solubility of various class II or class IV drugs.

\section{References}

1. Manda P, Hargett JK, Vaka SR, Repka MA, Murthy SN (2011) Delivery of cefotaxime to the brain via intranasal administration. Drug Dev Ind Pharm 37: 1306-1310.

2. Manda P, Sammeta SM, Repka MA, Murthy SN (2012) lontophoresis across the proximal nail fold to target drugs to the nail matrix. J Pharm Sci 101: 23922397

3. Manda P, Angamuthu M, Hiremath SR, Raman V, Murthy SN (2014) Iontophoretic drug delivery for the treatment of scars. J Pharm Sci 103: 16381642.

4. Yang Y, Manda P, Pavurala N, Khan MA, Krishnaiah YS (2015) Development and validation of in vitro-in vivo correlation (IVIVC) for estradiol transdermal drug delivery systems. J Control Release 210: 58-66.

5. Xu X, Al-Ghabeish M, Rahman Z, Krishnaiah YS, Yerlikaya F (2015) Formulation and process factors influencing product quality and in vitro performance of ophthalmic ointments. Int J Pharm 493: 412-425.

6. Köllmer M, Popescu C, Manda P, Zhou L, Gemeinhart RA (2013) Stability of benzocaine formulated in commercial oral disintegrating tablet platforms. AAPS PharmSciTech 14:1333-1340. 
Citation: Dudhipala N (2016) A Review of Novel Formulation Strategies to Enhance Oral Delivery of Zaleplon. J Bioequiv Availab 8: 211-213. doi:10.4172/jbb.1000297

7. Manda P, Kushwaha AS, Kundu S, Shivakumar HN, Jo SB, et al. (2016) Delivery of ziconotide to cerebrospinal fluid via intranasal pathway for the treatment of chronic pain. J Control Release 224: 69-76.

8. Dudhipala N, Veerabrahma K (2016) Candesartan cilexetil loaded solid lipid nanoparticles for oral delivery: characterization, pharmacokinetic and pharmacodynamic evaluation. Drug Deliv 23: 395-404.

9. Dudhipala N, Veerabrahma K (2015) Pharmacokinetic and pharmacodynamic studies of nisoldipine-loaded solid lipid nanoparticles developed by central composite design. Drug Dev Ind Pharm 41: 1968-1977.

10. Dooley M, Plosker GL (2000) Zaleplon: a review of its use in the treatment of insomnia. Drugs 60: 413-445.

11. Drover DR (2004) Comparative pharmacokinetics and pharmacodynamics of short-acting hypnosedatives: zaleplon, zolpidem and zopiclone. Clin Pharmacokinet 43: 227-238.

12. Waghmare A, Pore Y, Kuchekar B (2008) Development and characterization of zaleplon solid dispersion systems: a technical note. AAPS PharmSciTech 9: 536-543.

13. Popescu C, Manda P, Juluri A, Janga KY, Cidda M, et al. (2015) Enhanced dissolution efficiency of zaleplon solid dispersions via modified ß-cyclodextrin molecular inclusion complexes. J Pharm Pharm Sci 1: 12-21.
14. Narendar D, Arjun N, Dinesh S, Karthik J (2016) Biopharmaceutical and Preclinical Studies of Efficient Oral Delivery of Zaleplon as Semisolid Dispersions with Self-emulsifying Lipid Surfactants. Int J Pharm Scin Nanotech 9: 1-8.

15. Janssens S, Nagels S, Armas HN, D'Autry W, Van Schepdael A, et at. (2008) Formulation and characterization of ternary solid dispersions made up of Itraconazole and two excipients, TPGS 1000 and PVPVA 64, that were selected based on a supersaturation screening study. Eur J Pharm Biopharm 69: 158-166

16. Damian F, Blaton N, Naesens L, Balzarini J, Kinget R, et al. (2000) Physicochemical characterization of solid dispersions of the antiviral agent UC781 with polyethylene glycol 6000 and Gelucire 44/14. Eur J Pharm Sci 10 : 311-322.

17. Janga KY, Jukanti R, Sunkavalli S, Velpula A, Bandari S, et al. (2013) In situ absorption and relative bioavailability studies of zaleplon loaded selfnanoemulsifying powders. J Microencapsul 30: 161-172.

18. Charman WN, Porter CJ, Mithani S, Dressman JB (1997) Physiochemical and physiological mechanisms for the effects of food on drug absorption: the role of lipids and pH. J Pharm Sci 86: 269-282.

19. Zakeri-Milani $P$, Valizadeh $H$, Tajerzadeh H, Azarmi $Y$, Islambolchilar Z, et al (2007) Predicting human intestinal permeability using single-pass intestinal perfusion in rat. J Pharm Pharm Sci 10: 368-379.

20. El-Shabouri MH (2002) Positively charged nanoparticles for improving the oral bioavailability of cyclosporin-A. Int J Pharm 249: 101-108. 